\title{
Guidance System to Target Spot for Charging by Communications
}

\author{
Naohisa Hashimoto*, Shin Kato*, Naoko Minobe*, Sadayuki Tsugawa**
}

\begin{abstract}
This paper is concerned with a docking assistance system on a charging station for electric vehicles (EV). An EV is automatically guided to a target docking spot for charging with high precision. This paper proposes a new concept of a vehicle guidance system for approaching a target spot for charging, which is an extension of the driver assistance by the cooperation between vehicles and infrastructure, and aims at an intelligent charging station. The system proposed here contributes to make it easy to approach a target spot for charging. Simulation studies and experiments are conducted to show the feasibility of the system proposed. In the experiment, a vehicle was guided by the proposed system that measures the position and the heading of a guided vehicle to indicate it the appropriate steering and the velocity to its goal.
\end{abstract}

\section{Keywords: Transportation Systems, Charging Station, Energy Supply, Guidance System, Communication System}

\section{INTRODUCTION}

There have been serious problems of air pollution, energy consumption, traffic accidents and congestion caused by automotive traffic. An electric vehicle (EV) contributes to solving the problems of air pollution, and energy consumption[1-3]. One of the most important problems in the penetration of EVs is the charging. It is important for an EV user that charging is speedier, easier and safer. In this study, we deal with an inductive type of charging. The inductive type has advantages that are less attrition and more safety. The inductive type of charging requires that an EV be positioned in a charging spot with high accuracy, but it can be usually achieved with guide rails at the spot. It is, thus, necessary that an EV must be guided to the spot with the high positioning accuracy. An essential problem in the inductive type of charging is, therefore, that an EV is guided to an optimal position at a charging spot.

The EV can be controlled to the target position for charging by using a driving or parking assistance system. Most driving assistance systems, however, are installed on a single vehicle, which will restrict the effects of the systems because the capability of onboard sensing systems is limited, and ordinary vehicles cannot afford a complete set of onboard sensing systems due to the cost and the room of the systems.

* National Institute of Advanced Industrial Science and Technology (AIST), AIST EAST 1-2-1, Namiki, Tsukuba-shi, Ibaraki, 301-8564, Japan, e-mail: naohisa-hashimoto@aist.go.jp shin.kato@aist.go.jp,n.minobe@aist.go.jp

** Meijo University / National Institute of Advanced Industrial Science and Technology (AIST), 1-501 Shiogamaguchi, Tempaku-ku, Nagoya-shi, Aichi, 468-8502, Japan, /AIST EAST 1-2-1, Namiki, Tsukuba-shi, Ibaraki, 301-8564, Japan e-mail: tsugawa@ccmfs.meijo-u.ac.jp
This paper proposes a new concept of a vehicle guidance system for approaching a target spot for charging, which is an extension of the driver assistance by the cooperation between vehicles and infrastructure [4-7], and aims at an intelligent charging station. This proposed charging system is one of the applications of automated vehicle guidance systems. Parking assistance or precision docking at loading zones have been proposed and verified. The system proposed here is an automated vehicle guidance system for EVs to a charging spot as shown in Fig.1.

This paper describes the concept of the system, algorithms of the measurement and the control, and reports some simulation and experimental results of the guidance assistance for a small EV.

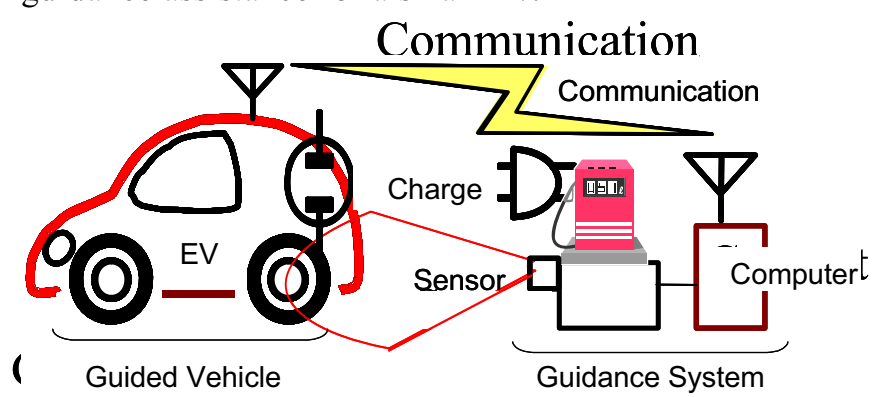

Fig. 1 Image of proposed system

\section{CONCEPT OF SYSTEM}

Figure 2 shows the general concept of the guidance system proposed here. We deal with an assistance system and an automated system. The system consists of a guidance or assistance system, and an automated or assisted vehicle. The communications between the 
guidance system and the guided vehicle play an essential role.

The proposed system has some levels of guidance, and variations of the configuration. Figure 3 illustrates two configurations: one (Fig. $3 \mathrm{a}$ ) is a driver assistance system, where a driver drives the vehicle with assistance from the guidance system, and the other (Fig.3b) is an automated guidance system, where the guided vehicle is automated and is automatically guided to its goal with the information from the guidance system.

In the system proposed here, the guidance system must be installed at a charging station. Thus, necessary sensors on the infrastructure can be of higher performance, more costly and larger than it on vehicle. Figure 4 shows the procedure from approaching to docking. The docking system fulfills the general requirements about charging system. When an EV reaches at the charging spot for connecting to plugs to the charging stand, the position demand requires very high accuracy that is under $5[\mathrm{~mm}]$. It is difficult for guidance system or to remotely guide the vehicle with this high degree of accuracy. Thus, when an EV approaches the charging spot, in other word after the guidance system guides the vehicle to the neighborhood of the desired spot, guide rail corrects the position error between a vehicle and the charging spot physically. As a result of studying on the requiring methods of proposed system by considering the characteristics of guide rail and mechanical shock, the necessary precision is under $0.2[\mathrm{~m}]$, and, proposed guidance system needs to have this precision. The proposed guidance system is constructed to achieve 0.2 [m] accuracy. In this paper, the guide rail which corrects the position for charging will not be described.

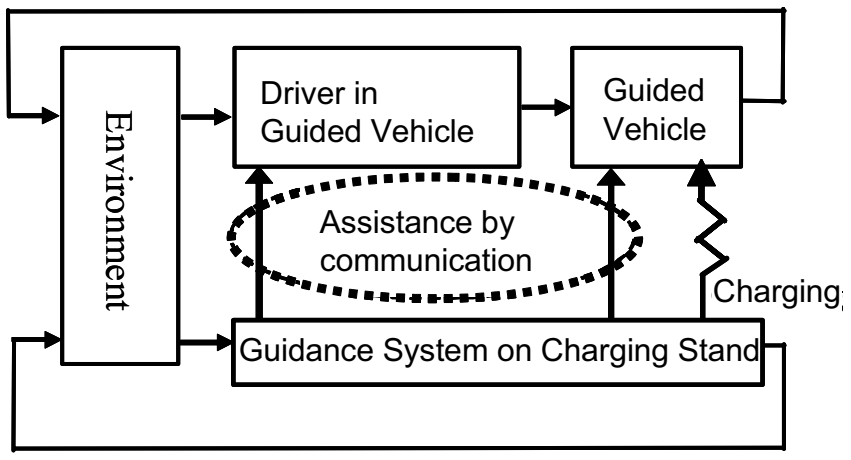

Fig.2 Concept of vehicle guidance system

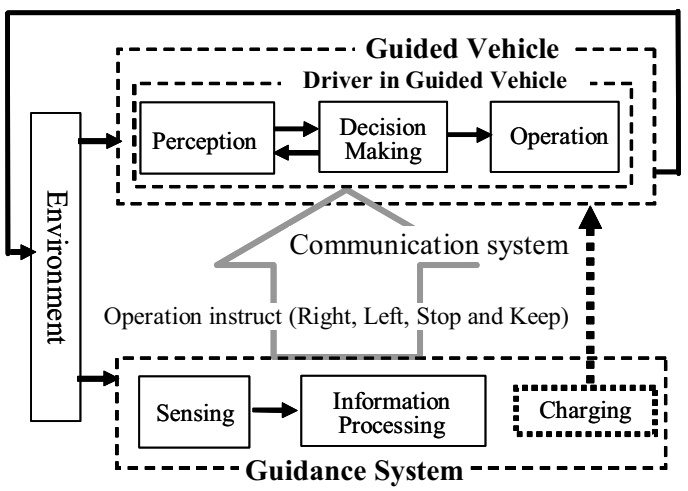

Fig.3a Several composition types of vehicle guidance system (Guidance system for driver)

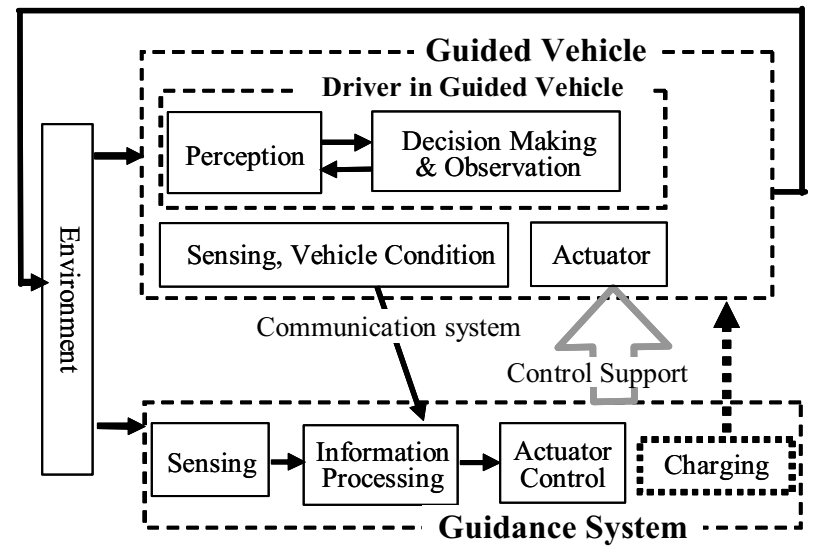

Fig.3b Several composition types of vehicle guidance system (Guidance system for automatically driving)

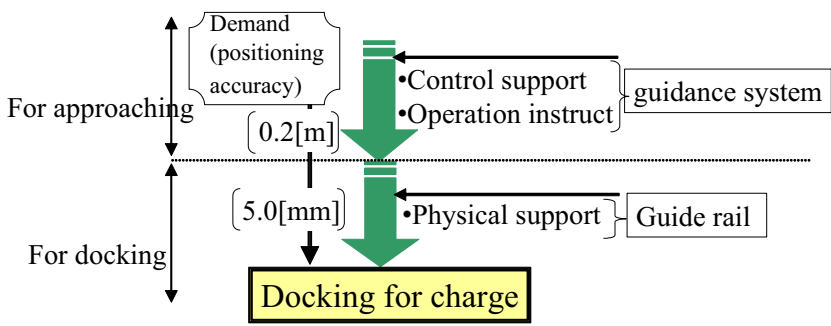

Fig.4 Demand position accuracy of approaching charging spot

\section{NECESSARY FUNCTIONS OF GUIDANCE TO TARGET SPOT FOR CHARGING}

\subsection{Overview}

This study focuses on guidance assistance to a target spot for charging, and will describe the method of detecting and estimating the state variables of a guided vehicle and the control algorithm. In this study, we assume that the guide rail will be employed and, thus, the requirement on the positioning accuracy of a vehicle is $0.2[\mathrm{~m}]$, instead of $5[\mathrm{~mm}]$.

The assistance will be conducted in the vicinity of a charging station with this system. The assistance starts when a guided vehicle arrives at a predetermined 
location. During the assistance, the guidance system measures the state variables of the guided vehicle, and calculates an appropriate steering and velocity for the guided vehicle to reach the goal or the planned target spot. The steering and velocity are transmitted over the communications. The guidance system is supposed to have the information on the geometry of the target spot and the initial location of the vehicle.

This guidance for an EV consists of the following steps:

1. Measurement of the state variables of an EV for control, and

2. Guidance control by considering the delay of communication.

3. The measurement and control are evaluated by experiment and simulation study.

\subsection{Measurement Algorithm of State Variables of Guided Vehicle for Control}

The algorithm to measure the state variables of a vehicle like the heading, the position, the yaw rate and the velocity is designed for the guidance assistance. A laser range-finder is used in this study. There are many kinds of sensors for measuring relative positions between an infrastructure device and a vehicle, for example, stereo vision, milliwave sensor and so on. In this paper, since our purpose is to investigate the feasibility, we employ a single sensor of a laser range-finder.

The laser range-finder measures the heading and the corners of the vehicle to calculate the center position of the vehicle by using its length and width. The measurement of the states of an EV including the yaw, the yaw rate, the position, and the velocity is conducted as follows:

The system searches the data from the laser range-finder and finds straight lines from the data,

The system selects two lines that cross each other with a right angle among the lines found,

The vehicle heading is calculated with the gradients of the two lines,

The point at the intersection of two lines is regarded as the vehicle's corner, and

The center position of the vehicle is found by use of the heading and the corner of the vehicle.

We deal with the center position instead of the center of gravity of a vehicle.

\subsection{Guidance Control Algorithm}

\subsubsection{Control Algorithm}

The necessary capability of the proposed system is that the system calculates optimal or feasible control to the vehicle for approaching the target spot based on the automated parking control algorithm by using the position and heading data of the vehicle, and that the system provides guidance information to the vehicle. The algorithm can be applied to two kinds of guidance: one is guidance assistance, and the other is automated control[8-13]. The algorithm is referenced to automated parking control algorithm[13]. The algorithm has characteristics that it does not depend on the parameters of the vehicle dynamics like the cornering power, the wheelbase or the tread. The algorithm, thus, is applicable to any vehicle and it is possible for our system to use this algorithm at very low speed which is proved by some experiments of the reference[13].

This study is premised on that the proposed system has the heading and position information of the target spot. The vehicle position is used for the center position of the vehicle in the algorithm in this study, because it is difficult to estimate the center of the vehicle of gravity. Figure 5 shows the steering control algorithm. The steering control algorithm is divided into 3 parts depending on the position and heading of a vehicle. The flow of the steering algorithm is as follows:

(i) If $\left.\left(P_{y}-V \cdot \sin \theta \cdot T_{d p}\right)>D_{t h} \quad \delta=K_{1} \mid \theta-0.5 \cdot \pi+\gamma \cdot\left(T_{p p}+T_{d p}\right)\right]$

$$
\text { If }\left(P_{y}-V \cdot \sin \theta \cdot T_{d p}\right) \leq D_{t h} \quad \delta=K_{2}\left\lfloor\theta+\gamma \cdot\left(T_{p p}+T_{d p}\right)\right\rfloor
$$

$$
\begin{aligned}
& \text { If }\left(P_{y}-V \cdot \sin \theta \cdot T_{d p}\right) \leq D_{t h} \cap\left\lfloor\theta+\gamma \cdot\left(T_{p p}+T_{d p}\right)\right\rfloor<A_{t h} \\
& \delta=K_{2}\left\lfloor\theta+\gamma \cdot\left(T_{p p}+T_{d p}\right)\right\rfloor+K_{3}\left\lfloor P_{y}-V \cdot\left(T_{p p}+T_{d p}\right) \cdot \sin \theta\right\rfloor
\end{aligned}
$$

where,

$D_{t h}$ : threshold of the starting yaw angle control [m], $A_{t h}$ : threshold of the starting lateral control [rad],

$T_{p p}$ : delay time of the control [s],

$T_{d p}$ : delay time of the communication [s],

$K_{1}, K_{2}, K_{3}$ : control gains, and

$\delta$ : target steering angle [rad].

In the velocity control method, the system keeps the vehicle at very slow speed during the guidance, and brakes the vehicle after the vehicle is in the vicinity of the target spot.

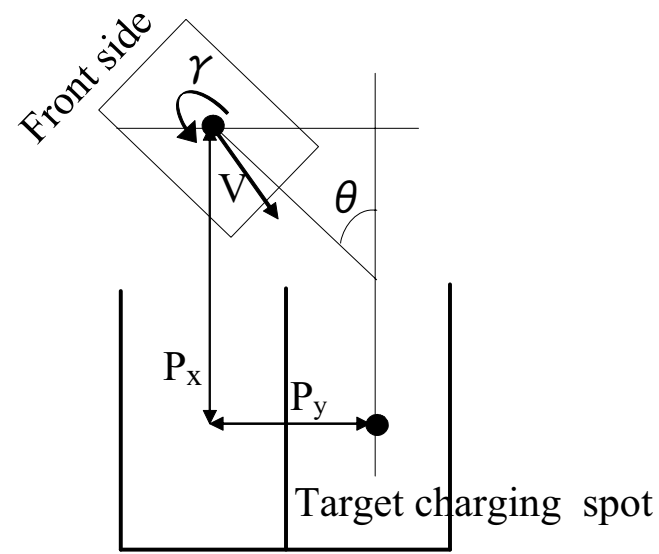

Fig.5 Guidance control algorithm

\subsubsection{Simulation of Guidance Control Algorithm}

The guiding control algorithm for the guidance assistance with the communications was also 
investigated by simulation studies. For the simulation, it is assumed that the proposed system estimates the vehicle state variables perfectly and the vehicle is controlled through the communications. The communication delay is set to 2.0 [s]. Figure 6 shows the simulation results. Several trajectories show that each vehicle is guided to the assigned spot. The simulation results show the feasibility that the proposed system is effective in guiding an EV to the target spot for charging.

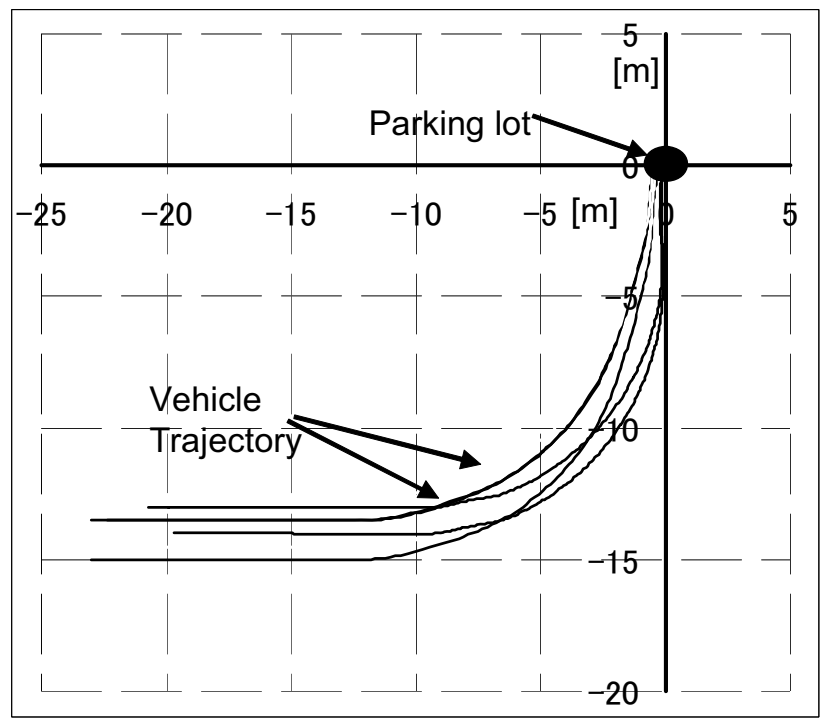

Fig.6 Simulation result (Vehicle Trajectory)

\section{EXPERIMENT OF AUTOMATED GUIDANCE WITH COMMNICATIONS}

The experiments are conducted by controlling the EV remotely and automatically through wireless-LAN communications, to verify the validity of the proposed guidance system.

\subsection{Outline and Configuration}

A laser range-finder is employed for the measurement as shown in Fig.7, and it provides distance data to an object within a direction of 180 degrees with 0.5 degree resolution. The laser sensor is set on the corner and in a same direction of the parking space at the height of $0.41[\mathrm{~m}]$, as shown in Fig.7. The arrow in Fig. 8 is the traveling direction of the vehicle. A laptop was used for the computation of the measurement. The parking lot, where the vehicle is guided to, is about $2.8[\mathrm{~m}]$ wide and $5.2[\mathrm{~m}]$ long. In this experiment, obstacles do not exist on the left side of the parking space and there is no blind area from the sensor to the vehicle. Figure 7 shows a setup of the proposed system. The wireless-LAN (2.4 [GHz]) system is used as communication device and it transmits data for control of the vehicle to the onboard computer with a period of 50 [msec].

In this experiment, in order to evaluate the position estimation and the remote control algorithm with the proposed system, RTK-GPS system (Novatel OEM4-G2) whose position accuracy is within 0.02 [m] is loaded on the vehicle. Although the measurement of the positions of a vehicle is not completely accurate due to the delay in the RTK-GPS, it is used as the reference data.

In the proposed system, the state variables of the guided vehicle are estimated with a laser range-finder and the target steering angle and velocity are calculated based on the automated parking control algorithm the control and, are sent to the vehicle through the wireless communications. When the proposed system calculates the amount of the target value, it uses the near future predicted vehicle state in consideration of delay of communications. The initial position of the vehicle is pre-determined by the system with some degree of freedom. After the system starts guidance, a driver does not need to control the vehicle and to be onboard the vehicle. The system configuration is shown in Fig.9. An ultra small electric test vehicle (EV) that has automated functions is used as a guided vehicle as shown in Fig.10. In this experiment, the test vehicle is remote-controlled, but a driver is in this vehicle for the safety reason.
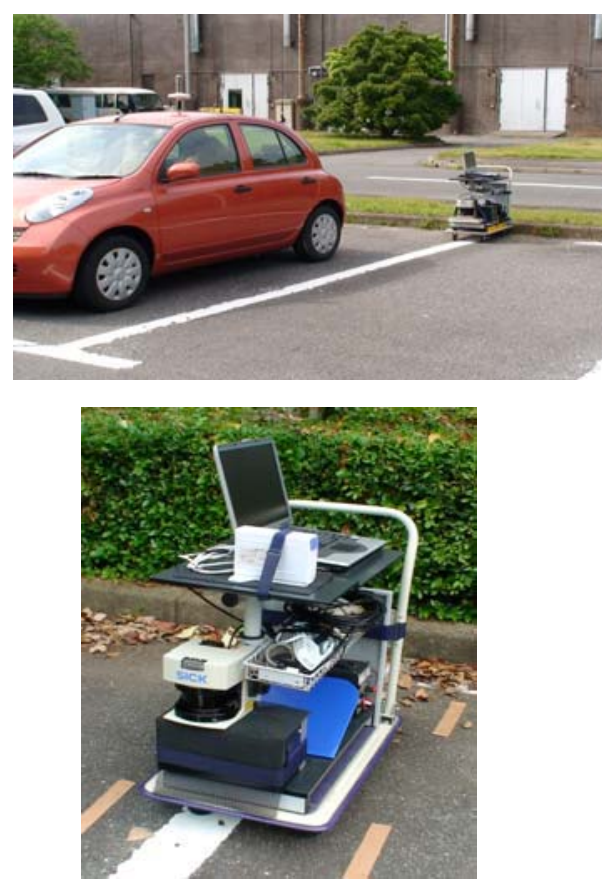

Fig.7 An experimental guidance system: (top) guidance system with parking lot, (bottom) a roadside guidance system 


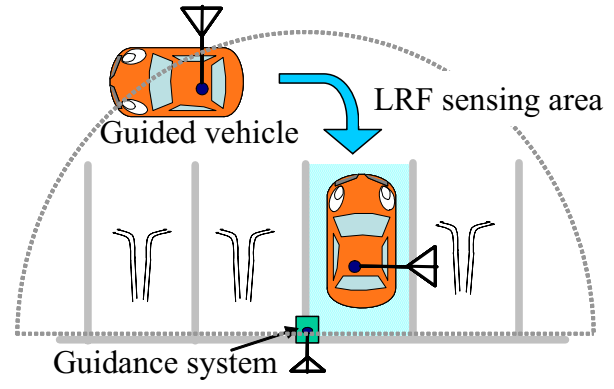

Fig.8 Experimental situation

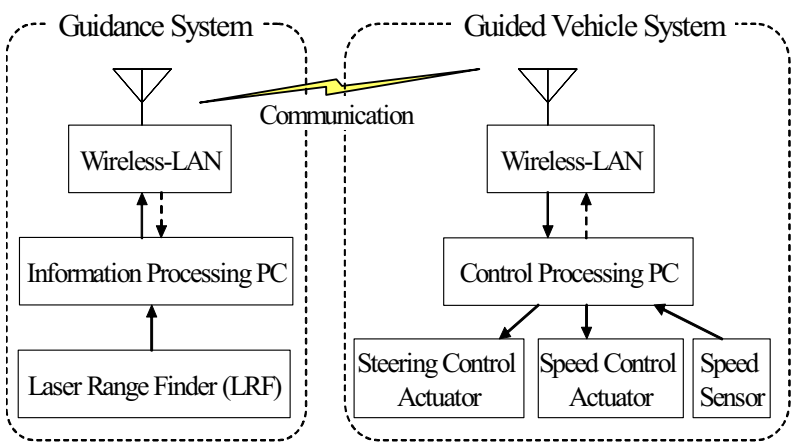

Fig.9 A system configuration when a road side guidance system has only a sensing function and a guided vehicle has automated actuating functions
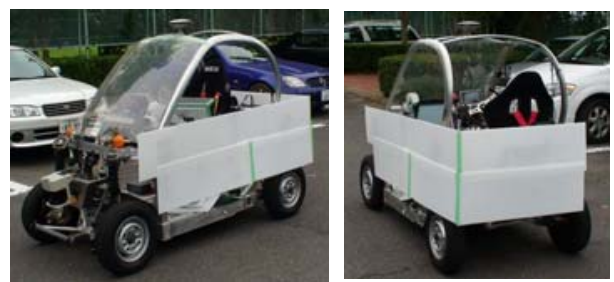

Fig.10 An ultra small electric test vehicle that has automated functions

\subsection{Experimental Results and Discussion}

The experimental results are shown in Figs.11, 12 and 13. Figure 11 shows two trajectories of the center of the guided vehicle; one is estimated by the proposed system and the other is measured by RTK-GPS. Figure 12 shows the error between the estimated positions by the system and those by the RTK-GPS based on the position that is regarded as absolute position. These results show the proposed system can estimate the vehicle's position without discontinuity.

Each error of the results is within about 0.2 [m] and, thus, the results suggest that the algorithm for the measurement of the state variables of the vehicle can estimate them without any discontinuity with enough accuracy for the vehicle guidance. The error of the estimated position is relatively large when the vehicle is far from the sensing system, but, it gets smaller as the vehicle approaches the sensing system. In addition, the $\mathrm{x}$ axis error shown in Fig. 12 is getting smaller as the vehicle approaches the sensing system. It is the error in the lateral displacement that must be kept small, and the result shows that the lateral error is small enough to guide a vehicle to the charging spot. The experimental result about error of the final target position is about 0.1 $[\mathrm{m}]$. Figure 13 shows the control values from the proposed system to the vehicle, where the minus value of the target velocity means a brake trigger from the system. The steering angle in Fig.13 indicates the control command of the proposed system, not the actual steering angle. Our control algorithm consists of 3 steps depending on the position of the vehicle to determine the steering angle. Thus, when the algorithm goes into next step the target steering angle is suddenly changed. In this experiment, the steering angle of the EV was changed not suddenly but steadily to the target steering angle. The reason why the target steering angle is fixed for a while is that the control command was above the maximum value to be set.

The vehicle is guided to the target space smoothly, automatically and with high accuracy. One of the features of the system is that a vehicle itself does not have any onboard sensors for the guidance.

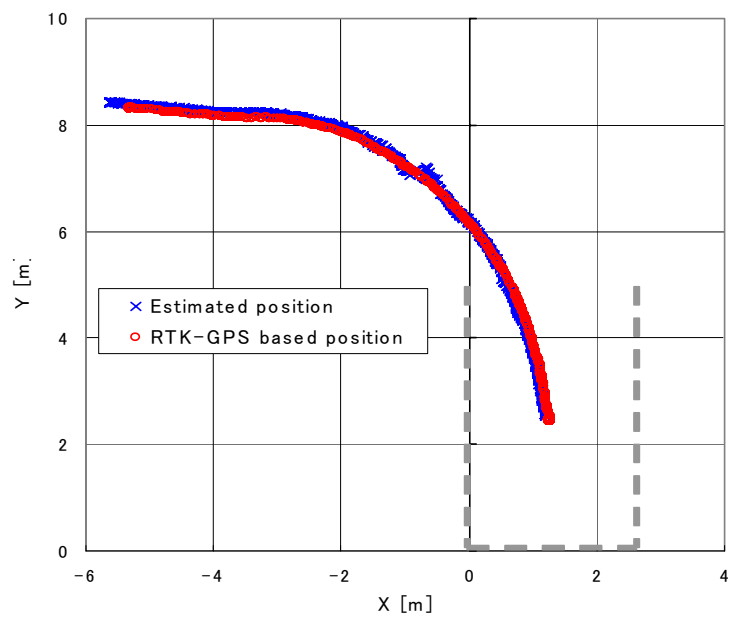

Fig.11 Vehicle trajectories measured with RTK-GPS and a roadside laser range-finder when the guided vehicle has automated functions

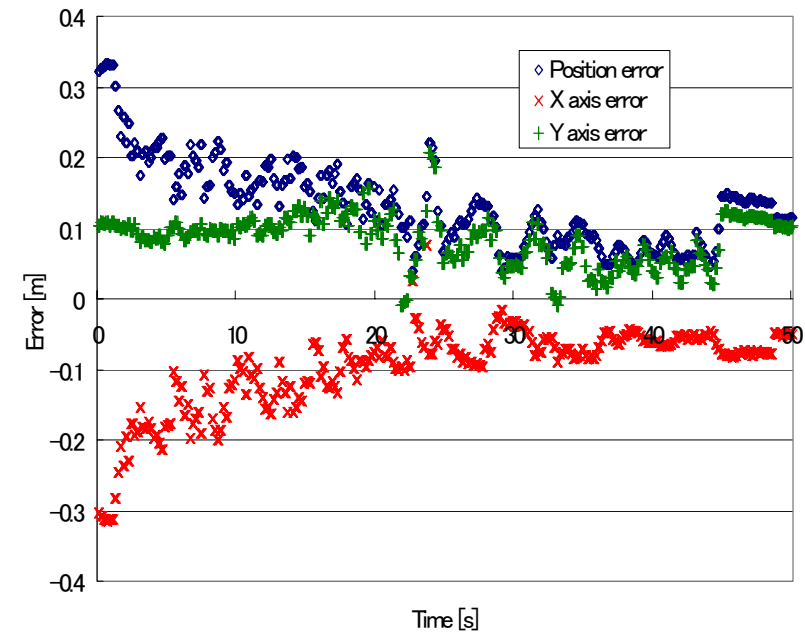

Fig.12 Measurement errors of vehicle locations when the reference is RTK-GPS measurements 


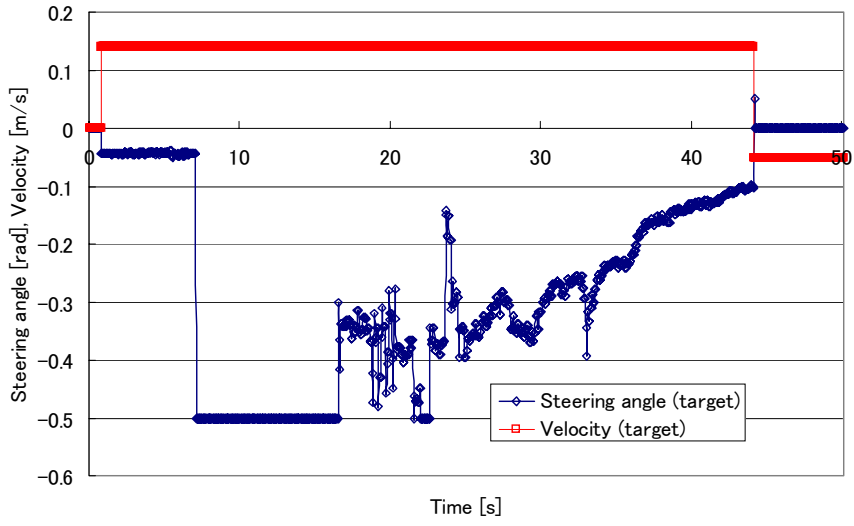

Fig.13 Steering and velocity commands from the roadside guidance system

\section{CONCLUSIONS}

A new concept that an EV is automatically guided to an assigned spot at a charging station has been proposed, and the experiments and the simulation studies have been conducted to show the feasibility of the system proposed.

Future work includes the evaluation of the proposed system with a real charging system which will be developed for the small electric vehicle, and useful, effective and comprehensive human machine interface (HMI) from the system to a driver for indication of vehicle control.

\section{ACKNOWLEDGEMENT}

This work has been supported by NEDO (New Energy and Industrial Technology Development Organization) since FY2002 under "Key Technology Research Promotion Program."

\section{REFERENCES}

[1] M. Nagai, et al., "Motion Control of Electric Vehicle by Distribution Control of Traction Forces", The Transactions of The Institute of Electrical Engineers of Japan, Series D, Vol.116-D, No.3, pp.279-284, 1996.

[2] K. Kawakami, et al., "Development of the Electric Lithium-ion Battery Car", The Transactions of The Society of Automotive Engineers of JAPAN, Vol.36, No.4, 2005.

[3] M.Kamachi, K.Walters : "A research of Direct Yaw-Moment Control on Slippery Road for In-Wheel Motor Vehicle", EVS22, CD-ROM, 2006.

[4] S. Kato, Y. Pan, S. Tsugawa : "Driver Assistance with Cooperative Driving", 7th International Symposium on Advanced Vehicle Control (AVEC04), CD-ROM, 2004.

[5] K. Matsumoto, H. Ota and T. Arai : "Multiple camera image interface for assisting in the control of mobile robots", Advanced Robotics, Vol.19, No.1, pp.39-53 2005.

[6] S. Kato, Y. Pan, S. Tsugawa : "A New Concept of Driver Assistance with Cooperation between Two Vehicles", 11th World Congress on Intelligent Transport Systems, CD-ROM, 2004.

[7] N. Hashimoto, S. Kato, N. Minobe, S. Tsugawa: "Autonomous Vehicle Guidance Through Cooperation Between Vehicle", ITS-world Congress, CD-ROM,
2006.

[8] S. E. Shladover: Review of the State of Development of Advanced Vehicle Control Systems(AVCS), Vehicle System Dynamics, Vol.24, No6-7, (1995), pp.551-595.

[9] M. Omae, N. Hashimoto, K. Shimoda, H Shimizu :"Development of Multi-purpose Small Electric Vehicle with Flat Floor and Drive-by-wire Configuration", EVS-19 CD-ROM, 2002.

[10] N. Hashimoto, M. Omae, K. Shimoda, H. Shimizu : "Automated Guiding Control System for Software Controlled Multi-purpose Small Electric Vehicle", EVS-20, CD-ROM, 2004.

[11] M. Omae, N. Hashimoto, T. Fujioka, H. Shimizu, "The Application of RTK-GPS and Steer-by-wire Technology to Automatic Driving of Vehicles and An Evaluation of Driver Behavior", Journal of International Association of Traffic and Safety Science (IATSS RESEARCH) Vol. 30, No. 2, pp.29-38, 2006.

[12] M. Omae, T. Fujioka: DGPS-Based Position Measurement and Steering Control for Automatic Driving, Proceedings of 1999 American Control Conference, pp. 3686-3690, 1999.

[13] M. Omae, N. Hashimoto, H. Shimizu: Automatic Driving System for Light Vehicle with Easy Setup Feature, Proceedings of the 21st International Electric Vehicle Symposium, 2005.

\section{BIOGRAPHIES}

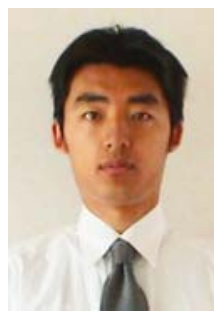

Naohisa Hashimoto is a researcher of intelligent system research division in National Institute of Advanced Industrial Science and Technology (AIST).

2002 Master's Degree Media and Governance from Keio University

2005 Ph.D Media and Governance from Keio University

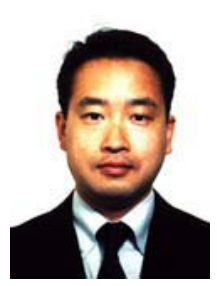

Shin Kato is a senior research scientist intelligent system research division in National Institute of Advanced Industrial Science and Technology (AIST)

1991 Master's Degree engineering from Meiji University

1994 Dr. Eng. degree from Meiji University

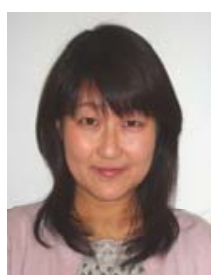

Naoko Minobe is a technical staff of intelligent system research division in National Institute of Advanced Industrial Science and Technology (AIST)

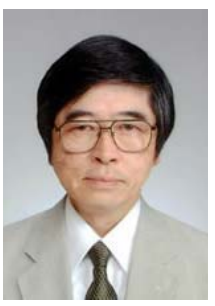

Sadayuki Tsugawa is a professor of Meijo University / National Institute of Advanced Industrial Science and Technology (AIST) 1970 Master's degree engineering from University of Tokyo 1973 Dr. Eng. degree from University of Tokyo 